\title{
Analyse de la dynamique végétale selon la nature et l'intensité du pâturage : exemple des marais communaux du marais poitevin
}

\author{
B. Amiaud, J.-B. Bouzillé, A. Bonis
}

Service d'écologie végétale, UMR 1853, Campus de Beaulieu, 35042 Rennes cedex, France

L'objectif de ce travail est l'analyse des scénarios de dynamique des communautés végétales des communaux du marais poitevin soumises à différentes pressions de pâturage par des bovins ou des équins. Un terrain de 20 ha est, depuis 3 ans, divisé en 10 enclos où s'exercent différents niveaux de chargement continu (de 0,6 à 2,4 UGB $\cdot$ ha $^{-1}$ ) d'avril à décembre. Chaque enclos comprend trois niveaux topographiques (dépressions inondables, pentes intermédiaires et replats supérieurs) qui constituent l'unité structurale du modèle agro-écologique de ce marais. La végétation des dépressions inondables est composée principalement de Glyceria fluitans et d'Eleocharis palustris. La végétation des bas de pentes intermédiaires comprend des espèces sub-halophiles comme Juncus gerardi et Hordeum marinum, alors que Carex divisa et Elymus repens dominent sur les parties hautes. La composition floristique des replats est caractérisée par Cynosurus cristatus et Gaudinia fragilis. Avec les deux types d'herbivores, une augmentation de la richesse spécifique (de une à dix espèces supplémentaires) est observée au sein de 24 groupements sur 28 analysés, pour les chargements moyens et forts. Au-dessus de 1,2 UGB·ha-1 ${ }^{-1}$ les communautés végétales de pentes intermédiaires s'étendent vers les replats supérieurs. L'impact des deux espèces d'herbivores apparaît cependant assez différent. Le pâturage par les équins crée une hétérogénéité plus marquée de la prairie avec l'existence de zones très rases ( 2 à $4 \mathrm{~cm}$ de hauteur) composées de plantes à rosettes telles que Plantago coronopus et Hypochaeris radicata situées au milieu d'une végétation plus haute où Elymus repens peut dominer.

\section{Impact du feu sur la valeur pastorale des landes de parcours dans les Pyrénées centrales}

\section{J. Faerber}

GEODE UMR 5602 CNRS, Université de Perpignan, 52, av. de Villeneuve, 66860 Perpignan cedex, France

La gestion des landes de parcours par des brûlages périodiques constitue dans les Pyrénées centrales une pratique traditionnelle qui s'est étendue au cours des dernières décennies aux terrasses de cultures abandonnées. Nous avons étudié l'évolution de composition floristique et de la phytomasse sur une vingtaine de parcelles ayant subi un feu courant dans des conditions contrôlées. La valeur pastorale (VP) a été calculée à partir des fréquences spécifiques relevées sur des lignes permanentes, corrigées en fonction de l'accessibilité et du recouvrement. Dans toutes les situations, le feu a permis une augmentation de la valeur pastorale dont l'ampleur et la durée dépendent du type de lande. Dans les landes à fougère-aigle (Pteridium aquilinum), l'augmentation reste faible et est limitée à 2-3 ans (VP 5-10 contre 1-5 à l'état initial). Dans les landes denses à genêt à balai (Cytisus scoparius), l'effet positif est plus important (VP 15-18 trois ans après le feu contre 0 à l'état initial); il peut persister plus de cinq ans si un pâturage intensif, tel que celui observé sur les passages obligés du troupeau, empêche la réinstallation des genêts. Dans les landes à callune (Calluna vulgaris) et autres Ericacées (Erica vagans, Vaccinium myrtillus...), l'augmentation considérable de la valeur pastorale (VP 15-18 six ans après le feu, contre 0,5 à l'état initial) peut être effective sur 8-10 ans, voire plus. Les résultats montrent que le feu est un outil intéressant pour la gestion des landes de parcours, en particulier dans le cadre d'une reprise de zones abandonnées. 